\title{
Comparison of SPECT bone scintigraphy with MRI for diagnosis of meniscal tears
}

\author{
Mohammad-naghi Tahmasebi*1, Mohsen Saghari ${ }^{\dagger 2}$, Masoud Moslehi ${ }^{\dagger 2}$ and \\ Ali Gholamrezanezhad ${ }^{\dagger 2}$
}

\author{
Address: ${ }^{1} \mathrm{MD}$, Orthopedic Department, Shariati hospital, Tehran University of medical sciences, Northern Kargar St. 14114, Tehran, Iran and ${ }^{2} \mathrm{MD}$, \\ Nuclear Medicine Research Center, Shariati hospital, Tehran University of medical sciences, Northern Kargar St. 14114, Tehran, Iran \\ Email: Mohammad-naghi Tahmasebi* - MNTahmasebi@hotmail.com; Mohsen Saghari - sagharim@sina.tums.ac.ir; \\ Masoud Moslehi - mmoslehi_m@yahoo.com; Ali Gholamrezanezhad - gholamrezanejhad@razi.tums.ac.ir \\ * Corresponding author †Equal contributors
}

Published: 14 April 2005

BMC Nuclear Medicine 2005, 5:2 doi:10.1186/147I-2385-5-2

This article is available from: http://www.biomedcentral.com/I47I-2385/5/2

(c) 2005 Tahmasebi et al; licensee BioMed Central Ltd.

This is an Open Access article distributed under the terms of the Creative Commons Attribution License (http://creativecommons.org/licenses/by/2.0), which permits unrestricted use, distribution, and reproduction in any medium, provided the original work is properly cited.
Received: 02 January 2005

Accepted: 14 April 2005

\begin{abstract}
Background: Scintigraphy has been considered as competitive to MRI, but limited data are available on the accuracy of single photon emission tomography (SPECT) compared with MRI for the assessment of meniscal tears. Our objective was to assess the value of SPECT in comparison to MRI.

Methods: Between January 2003 and March 2004, sixteen patients were studied with both modalities and the accuracy rates of SPECT scan results, and MRI findings in the diagnosis of meniscal tears were compared. Arthroscopy was the gold standard.

Results: The respective sensitivity rate, specificity rate, and positive and negative predictive accuracies of MRI were $89 \%, 94 \%, 93 \%$, and $79 \%$ and for SPECT those were $78 \%, 94 \%, 94 \%$, and $88 \%$. There was good agreement on the presence or absence of tears between two modalities $(\kappa$ statistic $=0.699)$.

Conclusion: SPECT and MRI are both valuable imaging techniques. SPECT is a useful alternative when MRI is unavailable or unsuitable and it is beneficial when more possible accuracy is desired (such as when MRI results are either inconclusive or conflict with other clinical data).
\end{abstract}

\section{Background}

In diagnosing meniscal tears, MRI is a sensitive and specific tool and has become the procedure of choice in these affections. In fact, MRI is the most commonly used noninvasive imaging method for diagnosing meniscal tears, but its limits are also acknowledged [1].

On the other hand, although it is not widely carried out in clinical practice, nuclear medicine procedures have also been used in diagnosing meniscal tears and some authors have demonstrated the usefulness of SPECT in the assessment of knee injuries [2-8].

In particular, recent data [9] have demonstrated a higher specificity and accuracy for 99mTc MDP SPECT than those for MRI. As a result, scintigraphy has been considered as competitive to MRI, and also it could give complementary informations which are commonly derived from scintigraphy but not available from MRI. 
There are not so much published works concerning meniscal tears and bone SPECT [10-14]. Only few studies have compared bone scintigraphy and MR imaging and limited data are available on the accuracy of SPECT compared with that of MRI for the assessment of meniscal tears. Therefore, it was emphasized that further work should be undertaken to evaluate the role of SPECT as a screening test for the evaluation of knee disorders.

This study reports the results of a recent prospective evaluation of MRI and SPECT bone scintigraphy and compares them with the results of arthroscopy as the gold standard test for the diagnosis of meniscal tears.

\section{Methods \\ Patients}

Between January 2003 and March 2004 sixteen consecutive patients ( 13 men, 3 women), aged $15-52$ yr ( $31 \pm 10$ $\mathrm{yr}$, Mean \pm S.D.), who were referred to our orthopedic surgeon were entered in this prospective study. Subjects were selected on the basis of positive history and clinical signs suggestive of meniscal tears. MRI and SPECT bone scintigraphy of both knees were obtained from all subjects. The time interval between the SPECT and MRI examinations was 1 to 3 weeks (mean time interval, 2.4 weeks). None of the patients had trauma or additional invasive therapeutic interventions between the SPECT and MRI scans. Patients also underwent arthroscopy of the affected knees.

\section{MRI}

All studies were performed using a scanner (IGE Medical Systems, Signa Herza, Milwaukee, WI) with a 1.5 Tesla magnet. The knees were placed in an extended position with approximately $15^{\circ}$ of external rotation. The imaging protocol included sagittal multiecho (repetition time msec/echo time msec, 2,500-3,600/20-120), coronal T1weighted (600/12), coronal multiecho $(2,500-3,000 / 17$ $119)$, and transverse gradient-echo or turbo T2-weighted sequences with a slice thickness of $4.5 \mathrm{~mm}$, no interslice gap, and a matrix of $256 \times 256$. MRI results were reported by a radiologist experienced in MRI of the knees.

\section{Bone scintigraphy}

A commercial MDP preparation (Myoview; Amersham International) was used. The labeling and quality control procedures were performed according to the manufacturer's instructions.

Scans were performed on a Vertex dual head ADAC camera. All patients received $750 \mathrm{MBq}(20 \mathrm{mCi}){ }^{99 \mathrm{mTc}}$ methylene diphosphonate ( $99 \mathrm{mTc}-\mathrm{MDP}$ ) by injection, and 3 hours later, anterior, posterior, medial and lateral views of both knees were obtained. SPECT was performed after securing the knees with a band around the tibiae and the legs straightened, with the same dual-head gamma cam- era, equipped with a pair of low energy, high resolution collimators. Images were acquired in a $128 \times 128$ matrix at 64 steps, $40 \mathrm{~s}$ each step. Data were processed by back projection and filtered by Hanning 0.8 filter. Images were reconstructed and displayed in all three orthogonal planes. Two experienced nuclear medicine physicians familiar with knee SPECT scans interpreted the findings of knee SPECT and the final diagnoses were reached by consensus. Blinded to other informations both MRI and bone SPECT were reported as definite or probable meniscal tear (positive) and normal or non-specific (negative). The positive criterion for meniscal tears was tibial plateau activity on the planar image with at least a half crescent of peripheral tibial plateau uptake on SPECT[12]. Other abnormal scintigraphic patterns were considered as non-specific. The positive criteria for meniscal tears in the MRI were abnormal morphology of the meniscus on one or more MR images and/or abnormal increased signal in that area on fat saturated proton density or T2-weighted images. Arthroscopy was performed by our experienced arthroscopist who already knows the results of the MRI and SPECT bone scans at the time of the examination.

\section{Statistical evaluation}

Results were analyzed on a per-meniscus basis. Using arthroscopy as a gold standard, the results of each modality were analyzed for sensitivity, specificity, negative predictive value and positive predictive value. Differences between these performance indices in the two modalities were evaluated with the McNemar test. $P<0.05$ was considered to be statistically significant.

All patients gave informed consent to participate in this study, which was approved by the committee on ethics at the faculty of medicine, university of Tehran.

\section{Results}

A total of 32 menisci, including 16 left and 16 right menisci, in 16 patients (table 1) were assessed. According to the arthroscopic results, tears were present in 18 (56\%) menisci (table 2), of these thirteen tears were in the medial menisci, five tears were in the lateral menisci, ten tears were in the left menisci and eight tears were in the right menisci.

By consensus, observers detected 15 meniscal tears at SPECT readings, of which one was falsely positive (table 3 ). One knee showed generalized increased uptake on bone SPECT images, in which the exact anatomical location of the pathologic process could not be determined and this finding was categorized as false-negative for meniscal tear. Overall there were only four false-negative SPECT scans. 
Table I: Patients' characteristics and results of MRI, SPECT and Arthroscopy.

\begin{tabular}{cccccc}
\hline Patient no. & Sex & Age & MRI & SPECT & Arthroscopy \\
\hline I & M & 34 & LM & LM & LM \\
2 & M & 15 & NL & NL & NL \\
3 & M & 19 & RM & RM & RM \\
4 & F & 52 & LM & LM/LL & LM/LL \\
5 & M & 45 & RM & RM & RM \\
6 & M & 35 & LM & LM & LM \\
7 & M & 30 & LL & LL & LL \\
8 & F & 50 & RM & D.I.U & RM \\
9 & M & 26 & RM/RL & RL & RM/RL \\
10 & M & 24 & RM & RM & RM \\
11 & M & 26 & LM & LM/LL & LM \\
12 & M & 28 & RM & RM & RM \\
13 & M & 25 & LL & NL & LM \\
14 & M & 30 & LM/LL & LM & LM/LL \\
15 & F & 32 & RL & RL & RL \\
\hline 16 & $M$ & 21 & LM & LM & LM
\end{tabular}

$* \mathrm{M}=$ Male, $\mathrm{F}=$ Female, D. I. U = Diffusely Increased Uptake, $\mathrm{RL}=$ Right Lateral, RM = Right Medial, LL = Left Lateral, LM = Left Medial, $\mathrm{NL}=$ Normal.

Table 2: Arthroscopy results by meniscus.

\begin{tabular}{cccc}
\hline Meniscus & Positive & Negative & Total \\
\hline Left/Medial & 7 & 1 & 8 \\
Left/Lateral & 3 & 5 & 8 \\
Right/Medial & 6 & 2 & 8 \\
Right/Lateral & 2 & 6 & 8 \\
\hline Total & 18 & 14 & 32 \\
\hline
\end{tabular}

Table 3: Bone SPECT readings by meniscus.

\begin{tabular}{cccc}
\hline Meniscus & Positive & Negative & Total \\
\hline Left & 9 & 7 & 16 \\
Right & 6 & 10 & 16 \\
\hline Total & 15 & 17 & 32 \\
\hline
\end{tabular}

MRI detected 17 tears (table 4). However one of them was false positive. Two meniscal tears were missed by MRI. Among the tears detected on SPECT images, only one tear was not detected by MRI. On the other hand, three meniscal tears were not depicted on SPECT images, which were detectable on MR images.
Table 5: A comparison of diagnostic ability of SPECT and MRI in diagnosis of meniscal tears.

\begin{tabular}{lcc}
\hline & MRI & SPECT \\
\hline Sensitivity & $89 \%$ & $\mathbf{7 8 \%}$ \\
Specificity & $94 \%$ & $\mathbf{9 4 \%}$ \\
Positive predictive value & $\mathbf{9 3 \%}$ & $\mathbf{9 4 \%}$ \\
\hline Negative predictive value & $\mathbf{7 9 \%}$ & $\mathbf{8 8 \%}$ \\
\hline
\end{tabular}

Table 4: MRI reading by meniscus.

\begin{tabular}{cccc}
\hline Meniscus & Positive & Negative & Total \\
\hline Left & 9 & 7 & 16 \\
Right & 8 & 8 & 16 \\
\hline Total & 17 & 15 & 32 \\
\hline
\end{tabular}

Table 6: Summary of knee SPECT and MRI results.

\begin{tabular}{lc}
\hline Test Results & No. of menisci \\
\hline Positive SPECT vs positive MRI & 13 \\
Positive SPECT vs Negative MRI & 2 \\
Negative SPECT vs Positive MRI & 4 \\
\hline Negative SPECT vs Negative MRI & 13 \\
\hline
\end{tabular}

Overall, MRI had a positive predictive value of $94 \%$ (16/ 17 ) and SPECT had 93\% (14/15). MRI had a negative predictive value of $88 \%(15 / 17)$ and SPECT had 79\% (15/ 19).

Table 5 shows the sensitivities, specificities, positive predictive values, and negative predictive values for each imaging modality. The two-tailed $p$ value of these differences equals 0.683 , which by conventional criteria, is considered to be not statistically significant. MRI and SPECT results were further compared on another per meniscus basis according to whether SPECT findings were positive or negative (table 6). In a total of 17 knees which underwent imaging with both modalities, meniscal tear was found in 17 menisci with MRI and in 15 menisci with SPECT. Concordant positive results were reported in 13 menisci. In four menisci, MRI depicted additional tears. The $\kappa$ value, as a measure for agreement between SPECT and MRI, revealed that despite differences between meth- 


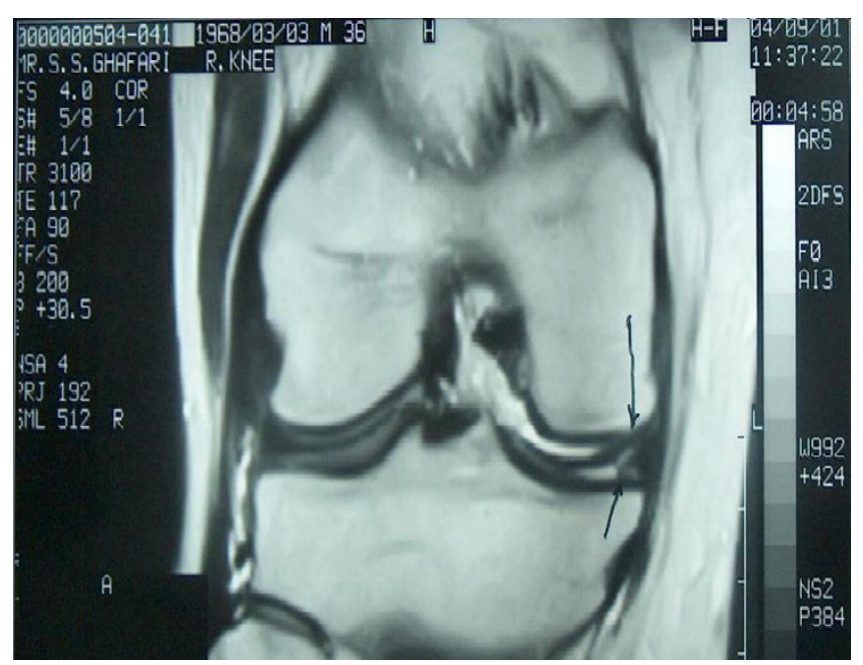

Figure I

Magnified coronal T2-weighted MRI image of the right knee showing a medial meniscal tear.

ods in sensitivity and specificity for the detection of meniscal tears, there was still good overall agreement $(\kappa$ statistic $=0.699$, with standard error $=0.101$ ).

\section{Conclusion}

MRI has become the radiologic procedure of choice for the diagnosis of meniscal tears. Recently SPECT also has been used for assessment of knee pathologies and has been documented to have a higher sensitivity than MRI. One of the most widely referenced studies is that of Ryan PJ et al. [9], in which 100 patients with undiagnosed knee pain were studied by clinical examination, MRI, SPECT bone scintigraphy and arthroscopy. The authors found the accuracy of MRI and SPECT in detecting meniscal tears to be comparable. Using arthroscopy as a gold standard, both MRI and SPECT showed high diagnostic ability to detect meniscal tears, with respective sensitivity rate, specificity rate, and positive and negative predictive accuracies of $80 \%, 71 \%, 84 \%$ and $71 \%$ for MRI and $84 \%, 80 \%$, $88 \%$ and $76 \%$ for SPECT. Some meniscal tears were detected by MRI alone $(n=5)$, or SPECT alone $(n=8)$. These authors concluded that SPECT bone scintigraphy is a suitable alternative to MRI to detect meniscal tears. It was also noted that the comparable diagnostic ability of SPECT bone scintigraphy implies that it can be used successfully when MRI is unavailable or unsuitable.

Even-Sapir and colleagues reported on 94 patients with suspected ACL/meniscal tear, or both who underwent SPECT followed by arthroscopy $(\mathrm{n}=74)$, magnetic resonance imaging $(n=37)$, or both [15]. Tears of the medial meniscus were diagnosed by arthroscopy in 43 patients. SPECT images detected increased uptake in the medial tibial plateau with a positive predictive value of $78 \%$ and a negative predictive value of $83 \%$. These authors suggest that bone SPECT is valuable in acute knee trauma for assessment of ACL, meniscal tears or both and for detection of associated bone injury.

In another study of patients with chronic knee pain Collier et al., found a high sensitivity of SPECT for the detection of meniscal tears, although specificity was less good [16].

In one of the initial studies, Murray et al. [11] found a SPECT sensitivity of $88 \%$ and specificity of $87 \%$ in patients with acute knee pain. They concluded that with respect to meniscal tears a negative bone scan can obviate the need for arthroscopy.

In the recent study done by Vellala RP et al. [17] the role of SPECT bone scan for the diagnosis of knee lesions in routine clinical practice was evaluated. Fourty consecutive case records were examined in patients who underwent a SPECT scan prior to knee arthroscopy in routine clinical practice. The accuracy of clinical examination, SPECT scan results, and arthroscopic findings (as the gold standard) in diagnosing knee lesions were compared. The sensitivity of SPECT scans in detecting medial meniscal and lateral meniscal lesions was $77 \%$ and $14 \%$, respectively. The specificities for the same structural lesions were high at $89 \%$ and 94\%, respectively. The authors concluded that SPECT bone scan appears to be useful in the diagnosis of knee pathology in routine practice and in selecting patients for arthroscopy, especially most useful for the diagnosis of medial meniscal tears.

Our results are not very different from the above-mentioned researches. The present prospective study demonstrated that MRI was only slightly superior to SPECT for detection of meniscal tears. However, the difference did not reach statistical significance. In our study SPECT revealed the majority of lesions seen on arthroscopy and MRI. Also there was a tear that was missed by MRI but adequately diagnostic by SPECT. Similarly there were some tears in which SPECT was negative but MRI showed the tears. It is not yet well determined that why some tears are missed by one modality and are detected by the other. However, it seems that in the presence of high clinical suspicion and negative MRI results (as the primary modality), SPECT can be helpful with detecting MRI negative tears. Despite of this fact, the detection of more tears at SPECT compared with MRI, did not lead to altered decision for treatment (i.e. conservative vs. arthroscopic treatment). The major reason is that the difference in detection of lesion was predominantly in patients in whom another 


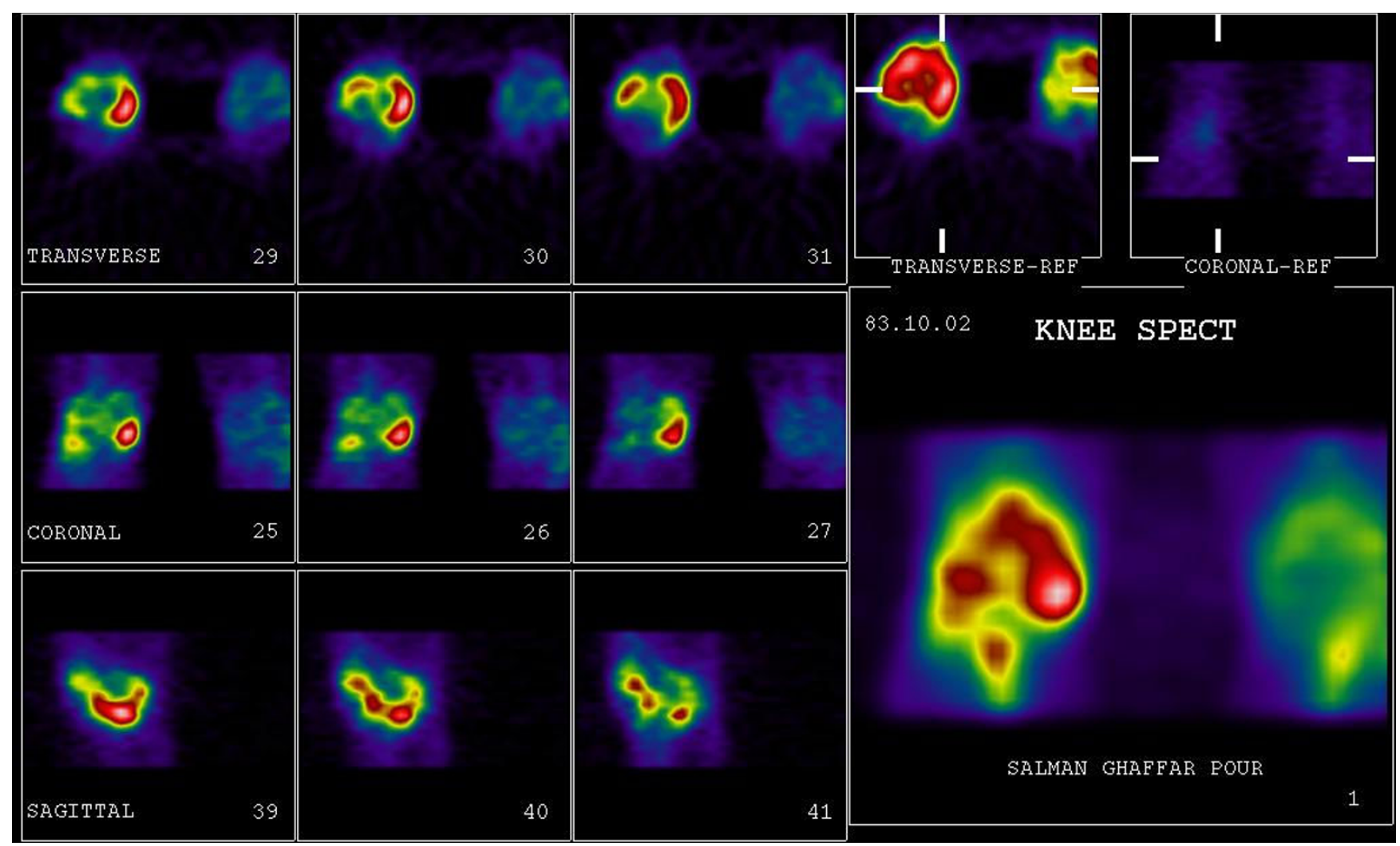

\section{Figure 2}

SPECT images of the same patient (presented in Figure I.) showing a crescent of increased activity in the medial tibial plateau, which is scintigraphically characteristic feature of a meniscal tear.

MRI-positive tear was detected. These patients are usually candidate for arthroscopy and therefore differences in the number of tears do not influence management.

In general, the findings of our study and that of previous studies suggest that examination with SPECT as well as with MRI can be used as a basis for the assessment of patients suspected for meniscal tears. However, both MRI $\&$ SPECT have various relative advantages and disadvantages. In general, SPECT is less costly than MRI because it involves lower capital equipment costs. SPECT is also widely available. The major limitation with the use of SPECT is the radiation exposure, the potential harm of which is poorly understood.

MRI also has some advantages over SPECT of the knees too. Most significantly, no ionizing radiation is used. MRI also has some important drawbacks, however. In most regions it is a more expensive than SPECT and has more contraindications and scheduling difficulties. Some authors concluded that MRI, except in certain circumstances, is an expensive and unnecessary diagnostic test in patients with suspected meniscal and ACL pathology (may be due to many false positive MRI reports) $[18,19]$.

These facts in addition to all of the above-mentioned research results indicate that SPECT and MRI are both valuable advanced imaging techniques but the absence of radiation exposure may make MRI preferable for the workup of patients suspected of having meniscal tears. Therefore it seems that all patients suspected for meniscal tears can be evaluated with MRI. However, SPECT has clear advantages when more possible accuracy is desired when MRI results are either inconclusive or conflict with other clinical data (i.e. SPECT should be performed if MRI is negative but there are clinical evidences of meniscal tear). SPECT may be available alternative when MRI is unavailable or unsuitable. This approach must be addressed in larger series of patients and a larger prospective study is currently being performed to confirm these data and approach. 


\section{Competing interests}

The author(s) declare that they have no competing interests.

\section{Authors' contributions}

MNT participated in the design of the study and carried out the arthroscopies. MS participated in the interpretation of the scintigraphic results. MM participated in its design and coordination, supervised the acquisition process and participated in the interpretation of the scintigraphic results. AG supervised the acquisition process, interpreted the scintigraphic results, performed the statistical analysis and drafted the manuscript. All authors read and approved the final manuscript.

\section{Acknowledgements}

This study was carried out with the sponsorship of Tehran University of medical sciences. We are indebted to Dr. Mohammad Eftekhari, Dr. Armaghan Fard Esfahani, Dr. Davoud Beigi, and Dr. Babak Fallahi (Nuclear Medicine research center, Shariati Hospital) and Dr. Ali Radmehr (Department of radiology, Shariati Hospital) for their consultations throughout the investigation and their suggestions. Thanks are also extended to the technologists at our hospital (especially Mr. S. M. A. Abdollahzadeh) for data acquisition.

\section{References}

I. Rath E, Richmond JC: The menisci: basic science and advances in treatment. BrJ Sports Med 2000, 34(4):252-7.

2. Lorberboym M, Ami DB, Zin D, Nikolov G, Adar E: Incremental diagnostic value of $99 \mathrm{mTc}$ methylene diphosphonate bone SPECT in patients with patellofemoral pain disorders. Nucl Med Commun 2003, 24(4):403-10.

3. Yildirim M, Gursoy R, Varoglu E, Oztasyonar Y, Cogalgil S: 99mTcMDP bone SPECT in evaluation of the knee in asymptomatic soccer players. Br J Sports Med 2004, 38(I): 15-8.

4. Gupta SM, Foster CR, Kayani N: Usefulness of SPECT in the early detection of avascular necrosis of the knees. Clin Nucl Med 1987, I 2(2):99-102.

5. Chung HW, Kim YH, Hong SH, Kim SS, Chung JK, Seong SC, Kang HS: Indirect signs of anterior cruciate ligament injury on SPET: comparison with MRI and arthroscopy. Nucl Med Commun 2000, 2 I(7):65I-8.

6. Cook GJ, Fogelman I: Lateral collateral ligament tear of the knee: appearances on bone scintigraphy with single-photon emission tomography. Eur J Nucl Med 1996, 23(6):720-2.

7. Cook GJ, Ryan PJ, Clarke SE, Fogelman I: SPECT bone scintigraphy of anterior cruciate ligament injury. J Nucl Med 1996, 37(8): 1353-6.

8. al-Janabi MA: The role of bone scintigraphy and other imaging modalities in knee pain. Nucl Med Commun 1994, I 5( I 2):991-6.

9. Ryan PJ, Reddy K, Fleetcroft J: A prospective comparison of clinical examination, MRI, bone SPECT, and arthroscopy to detect meniscal tears. Clin Nucl Med 1998, 23(I 2):803-6.

10. Grevitt MP, Taylor M, Churchill M, Allen P, Ryan PJ, Fogelman I: SPECT imaging in the diagnosis of meniscal tears. J $R$ Soc Med 1993, 86(II):639-4I.

II. Murray IP, Dixon J, Kohan L: SPECT for acute knee pain. Clin Nucl Med 1990, I5(II):828-40.

12. Ryan PJ: Bone SPECT of the knees. Nucl Med Commun 2000, 2 I ( I 0):877-85.

13. So Y, Chung JK, Seong SC, Sohn YJ, Kang HS, Lee DS, Lee MC: Usefulness of $99 \mathrm{Tcm}-\mathrm{MDP}$ knee SPET for pre-arthroscopic evaluation of patients with internal derangements of the knee. Nucl Med Commun 2000, 2 I(I): 103-9.

14. Ryan PJ, Chauduri R, Bingham J, Fogelman I: A comparison of MRI and bone SPET in the diagnosis of knee pathology. Nucl Med Commun 1996, I7(2): |25-31.
15. Even-Sapir E, Arbel R, Lerman H, Flusser G, Livshitz G, Halperin N: Bone injury associated with anterior cruciate ligament and meniscal tears: assessment with bone single photon emission computed tomography. Invest Radiol 2002, 37(9):52I-7.

16. Collier BD, Johnson RP, Carrera GF, Isitman AT, Veluvolu P, Knobel J, Hellman RS, Barthelemy CR: Chronic knee pain assessed by SPECT: comparison with other modalities. Radiology 1985 , I57(3):795-802.

17. Vellala RP, Manjure S, Ryan PJ: Single photon emission computed tomography scanning in the diagnosis of knee pathology. $J$ Orthop Surg (Hong Kong) 2004, I 2(1):87-90.

18. Rose NE, Gold SM: A comparison of accuracy between clinical examination and magnetic resonance imaging in the diagnosis of meniscal and anterior cruciate ligament tears. Arthroscopy 1996, I 2:398-405.

19. Terry GC, Tagert BE, Young MJ: Reliability of the clinical assessment in predicting the cause of internal derangement of the knee. Arthroscopy 1995, I I:568-76.

\section{Pre-publication history}

The pre-publication history for this paper can be accessed here:

http://www.biomedcentral.com/1471-2385/5/2/prepub
Publish with Biomed Central and every scientist can read your work free of charge

"BioMed Central will be the most significant development for disseminating the results of biomedical research in our lifetime. " Sir Paul Nurse, Cancer Research UK

Your research papers will be:

- available free of charge to the entire biomedical community

- peer reviewed and published immediately upon acceptance

- cited in PubMed and archived on PubMed Central

- yours - you keep the copyright

Submit your manuscript here:

http://www.biomedcentral.com/info/publishing_adv.asp
BiolMedcentral 